\title{
THE SPACES OF QUANTUM STATES FOR SOME HARMONIC OSCILLATOR POTENTIALS ON THE PUNCTURED PLANE C $\backslash\{0\}$
}

\author{
JAN MILEWSKI \\ Institute of Mathematics, Poznań University of Technology \\ ul. Piotrowo 3A, 60-965 Poznań, Poland \\ E-mail: jsmilew@wp.pl
}

\begin{abstract}
We consider equivariant solutions of Schrödinger equations on $\mathbf{C} \backslash\{0\}$ with harmonic oscillator potentials. We determine the spaces of equivariant quantum states in three cases: for an isotropic and anisotropic harmonic oscillator potential centered at 0 , and for a potential not centered at 0 .
\end{abstract}

1. Introduction. We deal here with a particular case of the topological quantum mechanics (TQM). The classical configuration space in TQM is non-simply connected [1], [2] and quantum states (or in other words wave functions) are suitable functions defined on the universal covering of the configuration space, or multivalued functions defined on the configuration space [3]-[5].

We consider the system whose configuration space is the punctured plane $\mathbf{C} \backslash\{0\}$. We study Hamiltonians with harmonic oscillator potentials. At first we consider the isotropic oscillator potential centered at the zero point. Using a suitable property of evolution operators we show that the space of equivariant quantum states in this case is the same as in the free particle case, so it is an infinite dimensional Hilbert space. Further we work with an anisotropic harmonic oscillator potential. In a similar way we show that the space of quantum states is the zero space. Finally we consider a harmonic oscillator potential centered at a point $z_{0} \neq 0$. Using a power resolution method we get that there is no equivariant solution of the stationary Schrödinger equation. This means that the space of equivariant quantum states of this Hamiltonian is also the zero space.

2000 Mathematics Subject Classification: 35J10, 81Q70.

Key words and phrases: topological quantum mechanics, space of quantum states, equivariant functions, evolution operator.

The paper is in final form and no version of it will be published elsewhere. 
The paper is organised as follows. In section 2 we study some properties of evolution operators of a free particle and harmonic oscillator on a straight line. In section 3 we give some information about TQM. In section 4 we show that the space of quantum states in TQM is not the space of all equivariant square integrable functions and we give a consistent definition of the quantum states. In sections 5-7 we determine the space of quantum states for harmonic oscillator potentials in the TQM.

2. Some properties of evolution operators and propagators. Now let us deal with some problems in quantum mechanics on a straight line. We identify the straight line with the real axis $\mathbf{R}$ and take the standard metric $d\left(x_{0}, x_{1}\right)=\left|x_{0}-x_{1}\right|$ for $x_{0}, x_{1} \in \mathbf{R}$. Then the Laplace operator is the second derivative $\Delta=\frac{d^{2}}{d x^{2}}$. The kinetic energy operator is

$$
T=-\frac{1}{2 m} \frac{d^{2}}{d x^{2}}
$$

we put here and in the sequel $\hbar=1$. To study the properties of evolution operators we use propagator as the integral kernel of evolution operator. In the following lemma we give some properties of integral kernels and evolution operators.

Lemma 1. Let the kernel of an operator $A$ be $K\left(x, x_{0}\right)$. Then

$$
K_{a, b}\left(x, x_{0}\right):=K\left(b x, a x_{0}\right)
$$

is the kernel of the operator

$$
A_{a, b}=a^{-1} d(b) \circ A \circ d\left(a^{-1}\right),
$$

where $d$ denotes the dilatation operator

$$
d(a) f(x)=f(a x) .
$$

We omit the elementary proof.

LEMMA 2. The evolution operator of the free particle of mass $m$ satisfies the following formula

$$
U(t)=d\left(\frac{1}{\sqrt{t}}\right) \circ U(1) \circ d(\sqrt{t}) .
$$

Proof. The propagator of a free particle [6]

$$
K_{0}\left(x, x_{0}, t\right)=\sqrt{\frac{m}{2 \pi i t}} \exp \left\{\frac{i m}{2 t}\left|x-x_{o}\right|^{2}\right\}
$$

satisfies

$$
K_{0}\left(x, x_{0}, t\right)=\frac{1}{\sqrt{t}} K_{0}\left(\frac{x}{\sqrt{t}}, \frac{x_{0}}{\sqrt{t}}, 1\right) .
$$

Using lemma 1 we get (5).

Lemma 3. The evolution operator $U_{\omega}$ of a particle of mass $m$ in the harmonic oscillator potential satisfies the following formula 


$$
\begin{aligned}
U_{\omega}(t)= & \sqrt{\frac{1}{\cos \omega t}} \exp \left\{-\frac{i m \omega}{2} \tan (\omega t) x^{2}\right\} \\
& \times d\left(\sqrt{\frac{\omega}{\sin \omega t \cos \omega t}}\right) \circ U_{0}(1) \circ d\left(\sqrt{\frac{\tan \omega t}{\omega}}\right),
\end{aligned}
$$

where $\omega$ is the frequency of the oscillator.

Proof. The Hamiltonian of the oscillator is

$$
H_{\omega}=T+\frac{1}{2} m \omega^{2}|x|^{2},
$$

where $\frac{1}{2} m \omega^{2}|x|^{2}$ is the potential. The propagator of the system is given by the formula $[6]$

$$
\begin{aligned}
K_{\omega}\left(x, x_{0}, t\right)= & \sqrt{\frac{m \omega}{2 \pi i \sin \omega t}} \\
& \times \exp \left\{\frac{i m \omega}{2 \sin \omega t}\left[\left(x^{2}+x_{o}^{2}\right) \cos \omega t-2 x x_{0}\right]\right\} .
\end{aligned}
$$

So the propagator $K_{\omega}$ can be expressed by means of $K_{0}$

$$
\begin{aligned}
K_{\omega}\left(x, x_{0}, t\right)= & \sqrt{\frac{\omega}{\sin \omega t}} \exp \left\{-\frac{i m \omega}{2} \tan (\omega t) x^{2}\right\} \\
& \times K_{0}\left(\sqrt{\frac{\omega}{\sin \omega t \cos \omega t}} x, \sqrt{\omega \cot \omega t} x_{0}, 1\right) .
\end{aligned}
$$

From lemma 1 we get (10).

3. Topological quantum mechanics. Let us consider a physical system with a nonsimply connected space $M$ of classical configurations. Let

$$
\chi: \pi_{1}(M) \rightarrow U(1)
$$

a unitary one dimensional representation of the fundamental group of $M$, and let

$$
L_{[\gamma]}: \widetilde{M} \rightarrow \widetilde{M}, L_{[\gamma]}(\widetilde{m})=L([\gamma], \widetilde{m})
$$

be the standard left free action of the fundamental group on the universal covering $\widetilde{M}$ [7]. A function $f: \widetilde{M} \rightarrow C$ is called $\chi$-equivariant if for any $[\gamma] \in \pi_{1}(M), \widetilde{m} \in \widetilde{M}$ the following equality

$$
f(L([\gamma], \widetilde{m}))=\chi([\gamma]) f(\widetilde{m})
$$

is satisfied.

Further we assume that the configuration space is a Riemannian space, and the covering is a Riemannian covering. This means that $\widetilde{M}$ is also Riemannian space and the covering projection

$$
p: \widetilde{M} \rightarrow M
$$

is a local isometry. So if $g$ ( $\widetilde{g}$ resp. $)$ is the metric on $M(\widetilde{M}$ resp. $)$ then

$$
\widetilde{g}=p^{*} g \text {. }
$$

We assume also that $d \mu$ is the Riemannian volume form on $M$. 
Let $f$ and $g$ be two $\chi$-equivariant functions. We see that the product $f \bar{g}$ is a $\pi_{1}(M)$ invariant function ( $\bar{g}$ denotes here the complex conjugation of $g$ ). So we can identify $f \bar{g}$ with a function defined on the base space $M$. Further by means of the identification we define the scalar product of $\chi$-equivariant functions by the formula

$$
\langle f, g\rangle=\int_{M} f \bar{g} d \mu
$$

where $d \mu$ is the measure on $M$. The space of quantum states in TQM is usually defined as the Hilbert space of square-integrable $\chi$-equivariant functions

$$
\mathcal{H}=L_{\chi}^{2}(M, \mathbf{C}, d \mu)
$$

with the scalar product (17). We show by an example in section 4 that this definition should be complemented by a physical condition connected with the evolution of a topological quantum system.

The kinetic energy operator of a one particle is

$$
T=-\frac{1}{2 m} \widetilde{\Delta}
$$

where $\widetilde{\Delta}$ is the Laplace operator on $\widetilde{M}$. The equality (16) gives

$$
\widetilde{\Delta}=p^{*} \Delta \text {. }
$$

Now let us consider a system whose configuration space is $\mathbf{C} \backslash\{0\}$. The fundamental group of the space is isomorphic to the additive group of integers

$$
\pi_{1}(M)=\mathbf{Z} .
$$

We define the unitary representation $\chi_{\nu}$ by the condition

$$
\chi_{\nu}(1)=e^{2 \pi i \nu},
$$

where $\nu \in[0,1)$ is the so called encircling parameter. The functions

$$
f_{1}(z)=z^{\nu} g_{1}\left(z, z^{*}\right), \quad f_{2}(z)=z^{* 1-\nu} g_{2}\left(z, z^{*}\right)
$$

are examples of $\chi_{\nu}$-equivariant functions, where $g_{1}, g_{2}$ are power series of the holomorphic and antiholomorphic variables with integer exponents, $z=x+i y, z^{*}=x-i y, x, y \in \mathbf{R}$, $x^{2}+y^{2}>0$.

\section{The Schrödinger equation and the problem of the space of quantum states} in TQM. Let us consider the Schrödinger equation on the punctured plane $\mathbf{C} \backslash\{0\}$

$$
H \Psi=i \frac{\partial \Psi}{\partial t}
$$

where the Hamiltonian $H$ is the sum of the kinetic energy operator

$$
T=-\alpha \frac{\partial^{2}}{\partial z \partial z^{*}}, \quad \alpha=\frac{2}{m}
$$

and a potential energy $V$. For $V=0$ we have a free particle system. Now we are going to give a definition of the space of quantum states in the TQM. For this purpose let us first consider the following example. 
EXAMPLE. Let

$$
\Psi\left(z, z^{*}, t\right)
$$

be an equivariant solution of the Schrödinger equation with equivariant initial condition $\Phi\left(z, z^{*}\right)$ for the free particle system:

$$
\Psi\left(z, z^{*}, 0\right)=\Phi\left(z, z^{*}\right), \quad T \Psi=i \frac{\partial \Psi}{\partial t} .
$$

So we can write

$$
\Psi\left(z, z^{*}, t\right)=U_{0}(t) \Phi\left(z, z^{*}\right),
$$

where $U_{0}(t)$ is the evolution operator of $H_{0}=T$.

Now let us modify the initial condition:

$$
\Psi_{a}\left(z, z^{*}, 0\right)=\exp \left[i\left(a^{*} z+a z^{*}\right)\right] \Phi\left(z, z^{*}\right) .
$$

Although the initial condition is also an equivariant one, the solution is not equivariant for $t \neq 0$ :

$$
\Psi_{a}\left(z, z^{*}, t\right)=\exp \left[i\left(-\alpha|a|^{2} t+a^{*} z+a z^{*}\right)\right] \Psi\left(z-\frac{a}{\alpha} t, z^{*}-\frac{a^{*}}{\alpha} t, t\right) .
$$

So we see that the space of equivariant functions is not invariant in relation to the evolution operator of the free particle system.

This example shows that we should reformulate the definition of the space of quantum states in TQM.

Definition. The Hilbert space of quantum states for a Hamiltonian $H$ is

$$
\mathcal{H}=\left\{\phi \in L_{\nu}^{2}(M, \mathbf{C}) \mid \forall t \in R: U(t) \phi \in L_{\nu}^{2}(M, \mathbf{C})\right\} .
$$

The restriction

$$
\bar{U}(t)=\left.U(t)\right|_{\mathcal{H}}
$$

is called the topological evolution operator of the quantum system.

5. Isotropic harmonic oscillator. In this section we consider the isotropic harmonic oscillator in $\mathbf{C} \backslash\{0\}$ with the Hamiltonian

$$
H_{\omega}=-\alpha \frac{\partial^{2}}{\partial z \partial z^{*}}+\frac{1}{2} m \omega^{2}|z|^{2} .
$$

THEOREM 1. The space of $\chi_{\nu}$-equivariant quantum states for the Hamiltonian $H_{\omega}$ is the same as for the free particle Hamiltonian $H_{0}=T$.

Proof. From the formula (10) we have that

$$
\begin{aligned}
U_{\omega}(t)= & \sqrt{\frac{1}{\cos \omega t}} \exp \left\{-\frac{i m \omega}{2} \tan (\omega t) x^{2}\right\} \\
& \times D\left(\sqrt{\frac{\omega}{\sin \omega t \cos \omega t}}\right) \circ U_{0}(1) \circ D\left(\sqrt{\frac{\tan \omega t}{\omega}}\right),
\end{aligned}
$$


where $D(a)$ is the homothety operator centered at 0 with scale $a, U_{0}(t)\left(U_{\omega}(t)\right.$ resp. $)$ is the evolution operator of TQM for the free particle (for the harmonic oscillator resp.). So the theorem follows from the next lemma.

LEMMA 4.

$$
f \in \mathcal{H}_{0} \Leftrightarrow \forall a \neq 0: D(a) f \in \mathcal{H}_{0} .
$$

Proof. Let

$$
f(z)=\sum_{l} \int_{0}^{\infty} f_{l, E} \Phi_{l, E}(z) d E
$$

be the spectral decomposition of the state $f$ for $H_{0}=T$, where $E$ is energy and $l$ is the repetition index. Then

$$
\begin{aligned}
& \left\|U_{0}(t) D(a) f\right\|^{2} \\
& \quad=\int_{M} \int_{0}^{\infty} \int_{0}^{\infty} \sum_{l l^{\prime}} \exp \left[-i a^{2}\left(E-E^{\prime}\right)\right] f_{l, E} \overline{f_{l^{\prime}, E^{\prime}}} \Phi_{l, E}(a z) \overline{\Phi_{l^{\prime}, E^{\prime}}(a z)} d E d E^{\prime} d \mu(z) \\
& \quad=\frac{1}{a^{2}} \int_{0}^{\infty} \sum_{l}\left|f_{l, E}\right|^{2} d E=\frac{1}{a^{2}}\|f\| .
\end{aligned}
$$

6. Anisotropic harmonic oscillator. The next example will be an anisotropic quantum oscillator on $\mathbf{C} \backslash\{0\}$. The Hamiltonian of the system is

$$
H_{\omega_{1}, \omega_{2}}=-\alpha \frac{\partial^{2}}{\partial z \partial z^{*}}+\frac{1}{2} m \omega_{1}^{2} x^{2}+\frac{1}{2} m \omega_{2}^{2} y^{2} .
$$

THEOREM 2. The space of $\chi_{\nu}$-equivariant quantum states for the Hamiltonian $H_{\omega_{1}, \omega_{2}}$ is the zero space.

Proof. From the formula (10) we get that the evolution operator for the system is

$$
\begin{aligned}
U_{\omega_{1}, \omega_{2}}(t)= & \frac{1}{\sqrt{\cos \omega_{1} t \cos \omega_{1} t}} \\
& \times \exp \left[-i \frac{m}{2}\left(\omega_{1}^{2} x^{2} \tan \omega_{1} t+\omega_{2}^{2} y^{2} \tan \omega_{1} t\right)\right] \\
& \times d_{x}\left(\sqrt{\frac{2 \omega_{1}}{\sin 2 \omega_{1} t}}\right) d_{y}\left(\sqrt{\frac{2 \omega_{2}}{\sin 2 \omega_{2} t}}\right) U_{0}(1) \\
& \times d_{x}\left(\sqrt{\frac{\tan \omega_{1} t}{\omega_{1}}}\right) d_{y}\left(\sqrt{\frac{\tan \omega_{2} t}{\omega_{2}}}\right)
\end{aligned}
$$

where $d_{x}\left(d_{y}\right.$ resp.) is the dilatation operator in the $x$ ( $y$ resp.) direction. Let $\Phi\left(z, z^{*}\right)=$ $z^{\nu} g\left(z, z^{*}\right)$ be a quantum state from $\mathcal{H}_{0}$. So $\Phi\left(z, z^{*}\right)$ belongs to the domain of the operator $U_{0}(1)$.

The product of operators $\left(d_{x}\left(\sqrt{\frac{\tan \omega_{1} t}{\omega_{1}}}\right)\right)^{-1}\left(d_{y}^{-1}\left(\sqrt{\frac{\tan \omega_{2} t}{\omega_{2}}}\right)\right)^{-1}$ deforms the variable $v=a x+i b y$ to $v_{t}=a \sqrt{\frac{\omega_{1}}{\tan \omega_{1} t}} x+i \sqrt{\frac{\omega_{2}}{\tan \omega_{2} t}} y$. For $a b>0$ the expression $v^{\nu}=(a x+i b y)^{\nu}$ is equivariant. Let $z_{t}=\sqrt{\frac{\omega_{1}}{\tan \omega_{1} t}} x+\sqrt{\frac{\omega_{2}}{\tan \omega_{2} t}} i y$. We see that the expressions $z_{t}{ }^{\nu}$ and 
$z_{t}^{* 1-\nu}$ are not holomorphic so $\Phi\left(z_{t}, z_{t}^{*}\right)$ is not a quantum state from $\mathcal{H}_{0}$. Further

$$
\left[d_{x}\left(\sqrt{\frac{\tan \omega_{1} t}{\omega_{1}}}\right) d_{y}\left(\sqrt{\frac{\tan \omega_{2} t}{\omega_{2}}}\right)\right]^{-1} \Phi\left(z, z^{*}\right)=\Phi\left(z_{t}, z_{t}^{*}\right)
$$

belongs to the domain of $U_{\omega_{1}, \omega_{2}}(t)$ because $d_{x}\left(\sqrt{\frac{\tan \omega_{1} t}{\omega_{1}}}\right) d_{y}\left(\sqrt{\frac{\tan \omega_{2} t}{\omega_{2}}}\right) \Phi\left(z_{t}, z_{t}^{*}\right)$ belongs to the domain of $U(1)$. This shows that the domain of $U_{\omega_{1}, \omega_{2}}(t)$ depends on time:

$$
\mathcal{D}\left(U_{\omega_{1}, \omega_{2}}(t)\right)=\left[d_{x}\left(\sqrt{\frac{\tan \omega_{1} t}{\omega_{1}}}\right) d_{y}\left(\sqrt{\frac{\tan \omega_{2} t}{\omega_{2}}}\right)\right]^{-1} \mathcal{D}(U(1)) .
$$

Moreover for a generic choice of $t_{1}, t_{2}$ we have

$$
\mathcal{D}\left(U_{\omega_{1}, \omega_{2}}\left(t_{1}\right)\right) \cap \mathcal{D}\left(U_{\omega_{1}, \omega_{2}}\left(t_{2}\right)\right)=\{0\} .
$$

This shows that

$$
\mathcal{H}_{\omega_{1}, \omega_{2}}=\{0\}
$$

REMARK. The domain of the evolution operator $U_{\omega_{1}, \omega_{2}}(t)$ depends on time, and the topological evolution operator $\bar{U}_{\omega_{1}, \omega_{2}}(t)$ is the operator with the zero domain.

7. Noncentral harmonic oscillator. The next example will be a noncentral harmonic oscillator. This means that the potential is centered at a nonzero point $z_{0}, V=\frac{1}{2} m \omega^{2} \mid z-$ $\left.z_{0}\right|^{2}$. In the sequel we put $\alpha=1, \frac{1}{2} m \omega^{2}=1$.

THEOREM 3. The space of equivariant quantum states for the Hamiltonian

$$
H_{z_{0}}=-\frac{\partial^{2}}{\partial z \partial z^{*}}+\left|z-z_{0}\right|^{2}
$$

is the zero space.

Proof. We consider the stationary Schrödinger equation

$$
-\frac{\partial^{2}}{\partial z \partial z^{*}} \Psi+\left|z-z_{0}\right|^{2} \Psi=E \Psi
$$

and we show that there is no solution of the eigenproblem. Let us use the following substitution

$$
\Psi=\exp \left(-\left|z-z_{0}\right|^{2}\right) \Phi
$$

So for $\Psi$ satisfying (44) the function $\Phi$ satisfies the following equation:

$$
-\frac{\partial^{2}}{\partial z \partial z^{*}} \Phi+\left(z-z_{0}\right) \frac{\partial}{\partial z} \Phi+\left(z^{*}-z_{0}^{*}\right) \frac{\partial}{\partial z^{*}} \Phi=\lambda \Phi,
$$

where $\lambda=E-1$. We look for a solution in the equivariant form:

$$
\Phi=\sum_{n m} A_{n m} z^{n+\nu} z^{* m}
$$

From the theory of quantum harmonic oscillator [8] we know that the solution $\Psi$ of the eigenproblem of the harmonic oscillator is a bounded function iff $\Phi$ in (47) is a finite sum of powers of $z$ and $z^{*}$. The equation (46) leads to the following birecursion equation for 
the coefficients $A_{n m}$ :

$$
\begin{aligned}
-(\nu+n) m A_{n m}=[ & \lambda-(n+m+\nu)+2] A_{n-1, m-1} \\
& +z_{0}(\nu+n) A_{n, m-1}+z_{0}{ }^{*} m A_{n-1, m} .
\end{aligned}
$$

Let $n_{0}$ be the greatest number such that $A_{n, m}=0$ for every $m$ and every $n<n_{0}$. Then

$$
\left(\nu+n_{0}\right) m A_{n_{0}, m}=z_{0}\left(\nu+n_{0}\right) A_{n_{0}, m-1},
$$

so for $z_{0} \neq 0$ and $\nu+n_{0} \neq 0$ the sum (47) has infinitely many summands. This shows that there is no $\chi_{\nu}$-equivariant bounded solution of the Schrödinger equation (44) for $z_{0} \neq 0$ and $\nu \in(0,1)$.

8. Concluding remarks. The space of quantum states in TQM depends on the choice of the Hamiltonian. The problem of quantum states was also discussed in [9]-[11]. In this paper we have shown that the space of equivariant quantum states for an isotropic harmonic oscillator on $\mathbf{C} \backslash\{0\}$ is the same as for the free particle system. In the paper [12] explicit formulas were given for propagators of these Hamiltonians for anyons systems. For $\omega$ tending to zero the propagator of the harmonic oscillator tends to the propagator of the free particle. So for the evolution operators we have the boundary condition

$$
\lim _{\omega \rightarrow 0} U_{\omega}(t)=U_{0}(t)
$$

in the TQM.

For Hamiltonians $H_{\omega_{1}, \omega_{2}}$ and $H_{z_{0}}$ the space of $\chi_{\nu}$-equivariant quantum states for $\nu \neq 0$ is the zero space. Also the limits of the topological evolution operators

$$
\lim _{\omega_{1}, \omega_{2} \rightarrow 0} \bar{U}_{\omega_{1}, \omega_{2}}, \quad \lim _{z_{0} \rightarrow 0} \bar{U}_{z_{0}}
$$

are the zero operators with the zero domain.

In traditional quantum mechanics $(\nu=0)$ the situation is different. The space of quantum states is the Hilbert space of square-integrable functions, and

$$
\lim _{\omega_{1}, \omega_{2} \rightarrow 0} U_{\omega_{1}, \omega_{2}}(t)=U_{0}(t) .
$$

Acknowledgements. The work has been supported by grant KBN No 1 P03A 03929.

\section{References}

[1] M. Laidlaw and C. DeWitt, Feynmann functionals integrals for systems of indistinguishable particles, Phys. Rev. D 3 (1971), 1375.

[2] J. S. Dowker, Quantum mechanics and field theory on multiply connected and homogeneous spaces, J. Phys. A 5 (1972), 936.

[3] F. Wilczek, Fractional Statistics and Anyons Superconductivity, World Sci., 1990.

[4] G. Morandi, The Role of Topology in Classical and Quantum Physics, Springer-Verlag, 1992.

[5] A. Lerda, Anyons Quantum Mechanics of Particles with Fractional Statistics, SpringerVerlag, 1992.

[6] R. P. Feynman and A. R. Hibbs, Quantum Mechanics and Path Integrals, McGraw-Hill, New York, 1965. 
[7] E. Spanier, Algebraic Topology, McGraw-Hill, New York, 1966.

[8] L. D. Landau and E. Lifshitz, Quantum Mechanics (Non-relativistic Theory), Pergamon, New York, 1977.

[9] J. Milewski and T. Lulek, The problem of the diagonal and the evolution operator in the theory of nnyons, Reports on Mathematical Physics 38 (1996), 279-282.

[10] J. Milewski, The Problem of completeness of states in topological quantum mechanics, Molecular Physics Reports 23 (1999), 171-176.

[11] J. Milewski, Equivariant functions and operators in topological quantum mechanics, J. Phys. Conf. Ser. 30 (2006), 182-187.

[12] J. Milewski and T. Lulek, Propagators of $N$ anyons on the plane $\mathbf{C}$, Reports on Mathematical Physics 59 (2007), 53-61. 
